\title{
Longitudinal Study of Inflammatory, Behavioral, Clinical and Psychosocial Risk Factors for Chemotherapy-Induced Peripheral Neuropath
}

lan Kleckner ( $\square$ ian_kleckner@urmc.rochester.edu )

University of Rochester Medical Center https://orcid.org/0000-0002-9828-9986

Todd Jusko

University of Rochester Medical Center

Eva Culakova

University of Rochester Medical Center

Kaitlin Chung

University of Rochester Medical Center

Amber S. Kleckner

University of Rochester Medical Center

Matthew Asare

Baylor College of Medicine

Julia E. Inglis

Liberty University

Kah Poh Loh

University of Rochester Medical Center

Luke J. Peppone

University of Rochester Medical Center

Jessica Miller

University of Michigan

Marianne Melnik

Cancer Research Consortium of West Michigan NCORP

Samer Kasbari

Southeast Clinical Oncology Research Consortium (SCOR)

Deborah Ossip

University of Rochester Medical Center

Karen M. Mustian

University of Rochester Medical Center 
Keywords: chemotherapy-induced peripheral neuropathy, CIPN, neuropathy, risk, inflammation

Posted Date: May 25th, 2021

DOI: https://doi.org/10.21203/rs.3.rs-544812/v1

License: (c) (1) This work is licensed under a Creative Commons Attribution 4.0 International License. Read Full License 


\section{Abstract}

Purpose. Chemotherapy-induced peripheral neuropathy (CIPN) is a common dose-limiting side effect of taxane and platinum chemotherapy for breast cancer. Clinicians cannot accurately predict CIPN severity partly because its pathophysiology is poorly understood. Although inflammation may play a role in CIPN, there are limited human studies. Here, we identified the strongest predictors of CIPN using variables measured before taxane- or platinum-based chemotherapy, including serum inflammatory markers.

Methods. 116 sedentary women with breast cancer (mean age 55 years) rated (1) numbness and tingling and (2) hot/coldness in hands/feet on 0-10 scales before and after six weeks of taxane- or platinumbased chemotherapy. A sub-study was added to collect cytokine data in the final 55 patients. We examined all linear models to predict CIPN severity at 6 weeks using pre-chemotherapy assessments of inflammatory, behavioral, clinical, and psychosocial factors. The final model was selected via goodness of fit.

Results. The strongest pre-chemotherapy predictors of numbness and tingling were worse fatigue/anxiety/depression (explaining $27 \%$ of variance), older age (9\%), and baseline neuropathy (5\%). The strongest predictors of hot/coldness in hands/feet were worse baseline neuropathy $(11 \%)$ and fatigue/anxiety/depression (6\%). Inflammation was a risk for CIPN, per more pro-inflammatory IL-1 $\beta$ (6\%) predicting numbness/tingling and less anti-inflammatory IL-10 predicting numbness and tingling (6\%) and hot/coldness in hands/feet (9\%).

Conclusions. The strongest pre-chemotherapy predictors of CIPN included worse fatigue/anxiety/depression and baseline neuropathy. A pro-inflammatory state also predicted CIPN. Because this is an exploratory study, these results suggest specific outcomes (e.g., IL-1ß) and effect size estimates for designing replication and extension studies.

\section{Trial Registration. NCT00924651}

\section{Background}

Over half of all patients with breast cancer receiving taxane- and platinum-based chemotherapy regimens experience chemotherapy-induced peripheral neuropathy (CIPN) [1, 2]. CIPN affects the hands and feet with sensory symptoms including numbness, tingling, and cold-induced, burning, and sharp-shooting pain; motor symptoms including cramping, difficulty handling small objects, and balance issues; and autonomic symptoms including dizziness [2,3]. CIPN is a dose-limiting toxicity, thereby potentially increasing mortality [4], and it interferes with activities of daily living and reduces quality of life. CIPN symptoms typically escalate over the weeks of chemotherapy [5] and recover slowly. Nearly half of all CIPN patients have symptoms six months after completing chemotherapy [2], and many require years to recover, if they recover at all [6]. Two key challenges in CIPN research are (1) clinicians cannot accurately predict which patients will experience the worst CIPN before initiating chemotherapy, and (2) there are very limited treatments for CIPN despite nearly 100 clinical trials in humans [7, 8], likely due to its poorly 
understood pathophysiology [9]. One way to help address these challenges is by conducting epidemiological studies to suggest (1) risk factors for CIPN and (2) mechanisms potentially related to the pathophysiology of CIPN, which can inform new interventions.

Previously implicated risk factors for CIPN that have been consistently observed across studies include pre-existing or early-onset symptoms of neuropathy (e.g., [10-14]), anxiety or depression (e.g., [13, 15]), and sedentary behavior (e.g., [16-18]); for a review see [2]. Some risk factors inconsistently observed across studies include older age (e.g., null findings in [10-12, 19]; positive findings in [16, 20]; opposite trend in [21]) and diabetes status (e.g., null findings in [12, 22, 23]; positive findings in [24]).

Although the pathophysiology of CIPN likely involves many factors [2, 9, 25], a growing body of evidence suggests that inflammation plays an important role [26-30]. Experimental studies in non-human animals have provided most of this evidence. For example, studies in mice show that pro-inflammatory cytokines (e.g., IL-1 $\beta$, tumor necrosis factor-a [TNF-a]) damage [31] affect the excitability [32] of peripheral nerves and thus lead to CIPN symptoms, such as hyperalgesia [32]. Another study in mice found that paclitaxel up-regulates inflammatory cytokines (IFN- $\gamma$, TNF-a, and others) in the spine concurrent with symptoms of CIPN [33].

Human research on inflammation and CIPN has been limited to a few case-control studies. A small study in 17 patients with breast cancer suggested that reduced IL- 6 before chemotherapy is associated with greater symptoms of CIPN after chemotherapy [34]. A study in 190 patients of mixed cancer types found that CIPN was more severe when anti-inflammatory drugs were withheld during infusion [35]. And finally, a study of 67 patients with breast cancer found that those with CIPN had markers of systemic inflammation (e.g., higher neutrophil-to-lymphocyte-ratio) compared to patients without CIPN [36]. Given that these studies are cross-sectional or small, additional studies are needed to investigate the relationship between CIPN and inflammation in humans.

The goals of this secondary analysis [37] are (1) to examine potential risk factors of developing CIPN using variables measured before chemotherapy, hopefully to assist clinicians in identifying who will experience CIPN before it occurs, and (2) to explore whether inflammation plays a role in CIPN in humans. We assessed patient-reported neuropathy symptoms before and after 6 weeks of neurotoxic taxane- or platinum-and taxane based chemotherapy in 116 female patients with breast cancer. The primary outcome for this study was numbness and tingling, and the secondary outcome was hot/coldness in the hands and feet. Potential risk factors were assessed before chemotherapy and include inflammation (serum cytokine concentration), clinical factors (chemotherapy type, cancer stage, body mass index [BMI], fatigue, anxiety, depression, use of diabetes medications), behavioral factors (physical activity), and demographic factors (age, race). We did not use a full CIPN questionnaire or assess pain because the parent study did not include a CIPN questionnaire or assessments of CIPN-specific pain (e.g., burning/shooting pain in the hands or feet). We hypothesized that more severe CIPN after 6 weeks of neurotoxic chemotherapy would be predicted by baseline (pre-chemotherapy) pro-inflammatory state (elevated IL-6, decreased IL-10), older age, worse fatigue, worse anxiety, worse depression, use of diabetes 
medications, and lower physical activity levels. This is an exploratory analysis that can help generate hypotheses, and inform outcomes and provide effect sizes for sample size calculations in future studies [37].

\section{Materials And Methods}

\section{Study design}

This was a secondary analysis [37] of patients with breast cancer randomized to usual care from a randomized clinical trial designed to assess the effects of exercise on fatigue among patients with cancer receiving chemotherapy (ClinicalTrials.gov NCT00924651; for details see [38-41]). The trial was conducted through the University of Rochester Cancer Center (URCC) National Cancer Institute (NCl) Community Oncology Research Program (NCORP) Research Base across 20 community oncology practices in the United States from 2009-2014. URCC and all community oncology institutional review boards approved the study before participants were enrolled. All participants provided written informed consent before beginning study assessments. All baseline measures were completed the week before randomization (week 0 ) and all final measures were obtained during week 6.

\section{Study participants}

To be eligible for the parent study, patients must have (1) been ${ }^{3} 21$ years old, (2) had a primary diagnosis of cancer other than leukemia, without distant metastasis, (3) been chemotherapy naïve, (4) started chemotherapy after enrollment and been scheduled for at least 6 weeks of chemotherapy with treatment cycles of either 2, 3, or 4 weeks long; (5) had a Karnofsky Performance Status ${ }^{370}$, (6) been able to read English, (7) not received concurrent radiation therapy, (8) not had physical limitations that contraindicate participation in a low- to moderate-intensity home-based walking and progressive resistance program (determined by the patient's oncologist, who had full knowledge of the program), and (9) not been identified as in the active or maintenance stage of exercise behavior as assessed by the Exercise Stages of Change [42]. This exploratory secondary analysis included all 116 patients with breast cancer randomized to usual care, received taxane- and/or platinum-based chemotherapy, and rated their neuropathy symptoms before and after 6 weeks of chemotherapy. We excluded patients randomized to exercise because this exercise intervention influenced multiple markers of inflammation (i.e., our assessed risk factors) [41].

\section{Measures}

Neuropathy symptoms were assessed via patient-reported numbness and tingling (primary outcome) and hot/coldness in hands/feet (secondary outcome) using 0-10 scales, where $0=$ "symptom not present" and 10 = "symptom as bad as you can imagine" during the last seven days. Validity and reliability have been demonstrated for similar scales $[15,43]$. The parent study did not include a full CIPN questionnaire or assessments of CIPN-specific pain. Patients were instructed to fast for morning blood draws and we used a Luminex MagPix (Austin, TX) to analyze concentrations of serum cytokines IL-1 $\beta$, IL-6, IL-8, IL-10, 
and IFN- $y$, and receptor STNFR1; for more detail see [41]. Measures of demographic and clinical factors were abstracted from medical records (chemotherapy type, cancer stage, and BMI) and patient report (age, race). We assessed fatigue using the multidimensional fatigue symptom inventory (MFSI) short form, which is a valid, reliable 30-item measure [44]. We assessed depression using the Center for Epidemiological Studies Depression Scale (CES-D) [45], a 20-item scale shown to reliably and validly measure depression in cancer populations [46]. We assessed anxiety using the Spielberger State/Trait Anxiety Inventory (STAI Form Y-1; i.e., only state anxiety) [47], a 20-item anxiety scale used in patients with cancer $[46,48]$. Physical activity was measured using a pedometer (Walk 4 Life Classic; Oswego, IL) worn on the waist for all waking hours of four days before the first chemotherapy infusion.

Cytokine data processing. All cytokine data were $\log _{10}$ transformed to reduce skew. We then removed 4 outliers that were greater than 3 standard deviations from the mean: 1 from $\log _{10}$ (sTNFR1), 1 from $\log _{10}(\mathrm{IL}-8)$, and 2 from $\log _{10}(\mathrm{IL}-10)$. Each cytokine had its own detection limit based on the measurement methodology. Nearly half the data were below the detection limit for IL-1 $\beta$ and IFN- $\gamma$ so they were set to categorical variables as described below.

Symptom cluster of fatigue, anxiety, and depression. In our bivariate analyses of potential risk factors, we observed a clustering of patient-reported fatigue, anxiety, and depression. Thus, we standardized each variable as a z-score and averaged the three standardized variables into a single composite score (Figure 1).

Statistical analyses. Analyses were performed using JMP software v.13 (SAS Institute Inc.; Cary, NC). Due to the exploratory nature of this work, we did not adjust for multiple comparisons to prevent Type II errors [49], i.e., incorrectly concluding that a true risk factor for CIPN is not associated with CIPN. To examine changes in CIPN, we used paired t-tests. To examine risk factors for CIPN, we used multiple linear regression to model post-intervention CIPN symptoms-(a) numbness and tingling or (b) hot/coldness in hands/feet-using an intercept term and all possible non-interaction combinations of risk factors. Our results describe the model with the lowest AICc (designated the final model) to balance competing goals of increasing goodness of fit and reducing the number of parameters [50]. In total, the potential risk factors were: baseline neuropathy symptoms (continuous; (a) numbness and tingling or (b) hot/coldness in hands/feet, to match the outcome), inflammation (where each cytokine had its own detection limit based on measurement methodology): serum concentration of IL-1 $\beta$ (categorical: not detected vs. low vs. high where low and high were determined via median split on detected values), IL-6 (continuous), IL-8 (continuous), IL-10 (continuous), IFN-ץ (detected vs. not detected), and sTNFR1 (continuous), clinical factors: neurotoxic chemotherapy type (categorical: only taxane vs. taxane and platinum), cancer stage (categorical: I vs. II, III, and IV), BMI (continuous), receiving diabetes medications (categorical: yes vs. no), composite score of z scores of fatigue, anxiety, and depression (continuous); behavioral factors: pedometer steps per day (continuous), and demographics: age (continuous), race (categorical: black vs. non-black). We reported the two final models (one for each CIPN outcome). 
We also report our analyses on two separate sub-cohorts: all patients $(N=116)$, and only patients who provided a blood specimen $(N=55)$. Blood collection only became available halfway through enrollment, and $89 \%$ of patients approached agreed to provide blood.

\section{Results}

\section{Baseline characteristics (Table 1)}

The 116 women with breast cancer were on average middle-aged (55 years), obese (BMI $30.3 \mathrm{~kg} / \mathrm{m}^{2}$ ), sedentary (4,412 steps/day), white (82\%), employed outside the house (55\%), married or in a committed relationship (66\%), and had at least some college education (67\%). Most cancer was early-to-mid stage (I, II, and III comprised 35\%, 53\%, and 10\%, respectively) with high Karnofsky Performance Status (59\% scored 100). Nearly all patients (91\%) had previously received surgery for their cancer, on average 6 weeks before starting chemotherapy. All patients received neurotoxic chemotherapy with the majority being only taxane-based (70\%), followed by combined taxane- and platinum-based (30\%).

\section{Severity and prevalence of neuropathy symptoms}

Before chemotherapy, the average severity of numbness and tingling was 1.14 and the average severity of hot/coldness in hands/feet was 0.92 on a 0-10 point scale (Table 2). After six weeks of neurotoxic chemotherapy, CIPN symptom severity significantly increased by 0.64 points $(95 \% \mathrm{Cl}: 0.17,1.11)$ for numbness and tingling and by 0.72 points for hot/coldness in hands/feet $(95 \% \mathrm{Cl}: 0.29,1.14)$. When dichotomizing CIPN symptoms as absent or present (per Bao et al., 2016; [15]), we observed an increase of $17 \%$ in prevalence of numbness and tingling from $36 \%$ to $53 \%$. For hot/coldness in hands/feet, we observed an increase of $16 \%$ in prevalence from $27 \%$ to $43 \%$. The same trends in severity and prevalence over time held in both sub-cohorts (Table 3).

\section{Assessment of potential risk factors excluding inflammation}

First, we examined the full cohort of 116 patients without the requirement for blood specimens to assess inflammation. The final model for numbness and tingling included worse fatigue/anxiety/depression ( $27 \%$ of variance), older age (9\%), worse baseline neuropathy (5\%), and black race (3\%). The final model for hot/coldness in hands/feet included worse baseline neuropathy ( $11 \%$ of variance), fatigue/anxiety/depression (6\%), and taxane chemotherapy (2\%). The model selection procedures consistently rejected several variables as potential risk factors for CIPN symptom severity: cancer stage, $\mathrm{BMI}$, use of diabetes medications, and pedometer steps (Figure 2).

\section{Assessment of potential risk factors including inflammation}

In all 55 patients who provided a blood specimen (Table 5), the final model for numbness/tingling included worse baseline neuropathy (28\%), stage I cancer (17\%), worse fatigue/anxiety/depression (15\%), higher pro-inflammatory IFN- $\gamma(12 \%)$, the use of diabetes medications $(11 \%)$, black race ( $8 \%)$, higher pro- 
inflammatory IL-1 $\beta$ (6\%), lower anti-inflammatory IL-10 (6\%) and higher pro-inflammatory IL-8 (1\%). The final model for hot/coldness in hands/feet included worse fatigue/anxiety/depression (26\%), higher proinflammatory IFN-y (18\%), worse baseline neuropathy (14\%), taxane chemotherapy (11\%), lower IL-6 (9\%), lower anti-inflammatory IL-10 (9\%), and lower BMI (6.5\%).

Taken together, these data suggest several pro-inflammatory markers that were significant risk factors for CIPN (IFN-y, IL-1ß, IL-8) and an anti-inflammatory marker that was protective against CIPN (IL-10), and IL6 , which was protective and has both pro- and anti-inflammatory properties [51]. These predictors each explained $1-18 \%$ of variance in the context of the other predictors in the final models. The potential risk factors for CIPN that were consistently rejected in our model selection procedure included sTNFR1, pedometer steps, and age (Figure 2).

\section{Discussion}

We explored several risk factors for predicting CIPN symptom severity after 6 weeks of neurotoxic taxane or platinum chemotherapy in women with breast cancer by assessing pre-chemotherapy inflammatory, behavioral, clinical and psychosocial risk factors. We found several consistent risk factors for CIPN including more severe baseline neuropathy, more severe fatigue/anxiety/depression, lower levels of antiinflammatory IL-10, and black race.

This is the largest and among the first study in human patients with cancer (not preclinical models, e.g. [33]) suggesting that a pro-inflammatory state before chemotherapy is a risk factor for CIPN. We found that lower levels of anti-inflammatory IL-10 and higher levels of pro-inflammatory IFN-y in serum were both risk factors for more severe CIPN, based on both patient-reports for the primary outcome numbness and tingling and the secondary outcome hot/coldness in hands/feet. Other risk factors included higher pro-inflammatory IL-1 $\beta$ (for numbness and tingling), pro-inflammatory IL-8 (for numbness/tingling), and lower IL-6 (for hot/coldness), which has both pro- and anti-inflammatory properties [51]. Our results are consistent with several prior studies: (1) one suggesting that blocking IL-1 $\beta$ signaling reduced CIPN symptoms in rats who received paclitaxel [52]; (2) a correlational study in humans suggesting that a single IL-1 $\beta$ polymorphism is a risk factor for CIPN [53]; and (3) a case-control study in humans suggesting perturbed gene expression in neuroinflammatory pathways in breast cancer survivors with CIPN vs. those without CIPN [54]. Our observations lend support and add specificity to theories that inflammation is involved in the etiology of CIPN in humans [26-28,55], and can help advance and inform anti-inflammatory treatments for CIPN $[56,57]$.

Our results suggest that one of the strongest risk factors for CIPN symptom severity is the symptom cluster of fatigue, anxiety, and depression (typically accounting for $18 \%$ variance), consistent with prior research $[13,15]$. These results invite the hypothesis that there is a shared biological mechanism of fatigue, anxiety, depression, and CIPN; the mechanism might be peripheral inflammation, neuroinflammation via disrupted blood-brain-barrier integrity [58], changes in the brain [59-61] including the interoceptive brain system, which processes bodily sensations [62], or mitochondrial dysfunction [63], 
all of which are implicated in the etiology of not only CIPN (for discussion, see [38]) but also fatigue, anxiety, and depression [63-65]. Furthermore, if fatigue, anxiety, and depression play causal roles in the development of CIPN symptoms, then interventions to reduce fatigue, anxiety, and depression could help prevent or alleviate symptoms of CIPN [66]. Potential interventions include (1) exercise, which improves depression [67], anxiety [67], fatigue [68], inflammation [69], and affects the interoceptive brain system (e.g., [70]), and may reduce CIPN [38, 39] (for a review, see [66, 71-73]); (2) pharmaceuticals such as duloxetine, which improves depression [74] and CIPN [75]; (3) neuromodulation or neurofeedback, which improves depression [76-78] and may also improve CIPN (e.g., [79]).

Our weakest observed risk factors for CIPN include age and the use of diabetes medications, consistent with mixed findings in the literature (see Introduction for citations). The mixed findings may be due to statistically weak associations and across-study differences in samples, sample sizes, use of a cutoff vs. continuous measures for age, and choice of other covariates. Some of the risk factors for CIPN that we observed might be due to confounding chemotherapy factors such as type and dose, which we did not collect. Physical activity (pedometer steps) and BMI were both weak or non-significant predictors of CIPN severity despite prior studies indicating that physical activity is protective [16-18] and BMI is a risk factor $[16,20]$. Our unexpected observations may be because our eligibility criteria ensured that our sample was sedentary (low steps, high BMI), thus restricting variability in physical activity levels. Our observation that black patients were at greater risk for CIPN severity was consistent with prior work [16], and is perhaps due to undiagnosed or untreated comorbidities such as prediabetes or diabetes, which is more prevalent in black Americans compared to white Americans [80-82].

This study has several strengths. First, to our knowledge, this is the largest longitudinal human study of inflammation and CIPN (116 patients, with 55 patients providing blood for inflammation measures; the only prior study involved 17 patients [34]). Second, assessing CIPN before and after six weeks of chemotherapy provides stronger evidence for CIPN risk factors than a cross-sectional post-chemotherapy design. Third, our data were obtained from community oncology clinics around the United States, enhancing the generalizability to our findings compared to single-site studies.

This study also has limitations. First, because this was an exploratory secondary analysis [37] based on subpopulation of 116 patients randomized to usual care from a randomized clinical trial, these findings should be tested for replication and further validation. With that said, our pattern of findings with pro- and anti-inflammatory cytokines are as expected based on prior literature and presumed mechanisms, lending credibility to these results. Second, the measures of CIPN were crude, with two 0-10 patient-reported questions instead of a full multi-question survey designed to assess CIPN. However, the measures we used have been successfully used and validated in prior studies (e.g., $[15,43])$. Moreover, we did not have access to clinical assessment of CIPN (e.g., monofilament test, nerve conduction) specific to CIPN. Third, we only measured the first 6 weeks of chemotherapy and therefore the severity of neuropathy in our sample was relatively low. However, our 6-week measure may serve as a surrogate for future CIPN severity, as paclitaxel causes a monotonic weekly increase in CIPN severity (e.g., Figure 4 from [83]). Fifth, our precision may be reduced given small cell sizes in some variables (e.g., only a few black patients). In 
addition, we do not have information on certain variables that might be related to neuropathy symptoms, such as smoking status, alcohol consumption, HIV status, and chemotherapy dose. Sixth, our assessment of serum cytokines may not necessarily reflect inflammation in the nervous system that contributes to CIPN. However, serum inflammation is convenient to assess and could serve as a useful biomarker of CIPN in the future. In addition, detection limits of cytokine assessment methodology forced us to use categorical coding for some cytokines (e.g., IL-1 $\beta$, IFN- $\gamma$ ). However, we still saw prediction of CIPN severity with cytokines that were not subject to categorical coding (e.g., IL-10, IL-6). Finally, it is not known how these results generalize to other populations, as our sample was primarily white, welleducated, female patients with breast cancer who were sedentary and reported mild (not severe) neuropathy.

\section{Conclusion}

In this exploratory secondary analysis involving 116 patients, the strongest risk factors for development of CIPN after 6 weeks of neurotoxic taxane or platinum chemotherapy included more severe baseline neuropathy and the symptom cluster of fatigue, anxiety, and depression. Our data in 55 patients also suggest that a pro-inflammatory state before chemotherapy is a significant risk factor for CIPN, making this the largest and among the first longitudinal studies of inflammation and CIPN in humans. Our findings invite the hypothesis of a common biological mechanism (e.g., inflammation, brain connectivity, mitochondrial dysfunction) that underlies the etiology of CIPN, as well as fatigue, anxiety, and depression. Given the exploratory nature of this work, future studies should test for replication. Ultimately, identifying the strongest predictors of CIPN can help clinicians inform patients of the risks of chemotherapy as well as identify and optimize promising mechanism-based treatments for CIPN.

\section{Declarations}

Funding. This study was funded by NIH/NCl U10CA037420, NIH/NCI UG1CA189961, NIH/NCI R25102618, NIH/NCI T32102618, and NIH/NCI K07CA221931 to I.R.K., NIH/NCI K99CA237744 to K.P.L., and NCORP supplement U10CA037420.

Conflicts of interest/Competing interests. The authors declare that they have no competing interests. I.R.K received consulting funds Charles River Analytics unrelated to this work. K.P.L. received consulting funds from Pfizer and Seattle Genetics unrelated to this work.

Availability of data and materials. The datasets used and/or analyzed during the current study are available from the corresponding author on reasonable request.

Code availability. Not applicable.

Authors' contributions. KMM designed and led the parent study. IRK designed and led this secondary analysis and wrote the manuscript with primary input from TAJ, EC, DJO, and KMM. All authors contributed to writing and editing the manuscript. All authors read and approved the manuscript. 
Ethics approval and consent to participate. URCC and all community oncology institutional review boards in the NCORP nationwide network who participated in this study approved the study before participants were enrolled. All participants provided written informed consent before beginning study assessments.

Consent for publication. Not applicable.

\section{Acknowledgements}

We thank the patients and research staff at the University of Rochester Cancer Center (URCC) National Cancer Institute (NCI) Clinical Oncology Research Program (NCORP) Research Base and all NCORP affiliates who contributed to the conduct of this study. We are especially grateful to Ann Colasurdo for her technical expertise in sample processing, and Ciara McGillivray for her help with the literature review.

\section{References}

1. Argyriou AA et al (2012) Chemotherapy-induced peripheral neurotoxicity (CIPN): an update. Crit Rev Oncol Hematol 82(1):51-77

2. Seretny $M$ et al (2014) Incidence, prevalence, and predictors of chemotherapy-induced peripheral neuropathy: A systematic review and meta-analysis. Pain 155(12):2461-2470

3. Postma TJ et al (2005) The development of an EORTC quality of life questionnaire to assess chemotherapy-induced peripheral neuropathy: the QLQ-CIPN20. Eur J Cancer 41(8):1135-1139

4. Lyman GH (2009) Impact of chemotherapy dose intensity on cancer patient outcomes. J Natl Compr Canc Netw 7(1):99-108

5. Reeves BN et al (2012) Further data supporting that paclitaxel-associated acute pain syndrome is associated with development of peripheral neuropathy: North Central Cancer Treatment Group trial N08C1. Cancer 118(20):5171-5178

6. Brouwers EE et al (2009) Persistent neuropathy after treatment with cisplatin and oxaliplatin. Acta Oncol 48(6):832-841

7. Hershman DL et al (2014) Prevention and management of chemotherapy-induced peripheral neuropathy in survivors of adult cancers: American Society of Clinical Oncology clinical practice guideline. J Clin Oncol 32(18):1941-1967

8. Loprinzi CL et al (2020) Prevention and management of chemotherapy-induced peripheral neuropathy in survivors of adult cancers: ASCO guideline update. J Clin Oncol 38(28):3325-3348

9. Dorsey SG et al., NCl Clinical Trials Planning Meeting for prevention and treatment of chemotherapyinduced peripheral neuropathy. J Natl Cancer Inst, 2019

10. Argyriou AA et al (2005) Peripheral neuropathy induced by administration of cisplatin- and paclitaxelbased chemotherapy. Could it be predicted? Support Care Cancer 13(8):647-651

11. Attal $\mathrm{N}$ et al (2009) Thermal hyperalgesia as a marker of oxaliplatin neurotoxicity: a prospective quantified sensory assessment study. Pain 144(3):245-252 
12. Dimopoulos MA et al (2011) Risk factors for, and reversibility of, peripheral neuropathy associated with bortezomib-melphalan-prednisone in newly diagnosed patients with multiple myeloma: subanalysis of the phase 3 VISTA study. Eur J Haematol 86(1):23-31

13. Lee KM et al (2018) Pre-treatment anxiety is associated with persistent chemotherapy-induced peripheral neuropathy in women treated with neoadjuvant chemotherapy for breast cancer. $J$ Psychosom Res 108:14-19

14. Molassiotis A et al (2019) Risk factors for chemotherapy-induced peripheral neuropathy in patients receiving taxane-and platinum-based chemotherapy. Brain behavior 9(6):e01312

15. Bao T et al (2016) Long-term chemotherapy-induced peripheral neuropathy among breast cancer survivors: prevalence, risk factors, and fall risk. Breast Cancer Res Treat 159(2):327-333

16. Greenlee $\mathrm{H}$ et al., BMI, Lifestyle Factors and Taxane-Induced Neuropathy in Breast Cancer Patients: The Pathways Study. J Natl Cancer Inst, 2017. 109(2)

17. Mols F et al (2015) Chemotherapy-induced peripheral neuropathy, physical activity and health-related quality of life among colorectal cancer survivors from the PROFILES registry. J Cancer Surviv $9(3): 512-522$

18. Stevinson C et al (2009) Physical activity in ovarian cancer survivors: associations with fatigue, sleep, and psychosocial functioning. Int J Gynecol Cancer 19(1):73-78

19. Argyriou AA et al (2006) Is advanced age associated with increased incidence and severity of chemotherapy-induced peripheral neuropathy? Support Care Cancer 14(3):223-229

20. Ghoreishi Z et al (2018) Risk factors for paclitaxel-induced peripheral neuropathy in patients with breast cancer. BMC Cancer 18(1):958

21. Greenwald MK et al., Risk of incident claims for chemotherapy-induced peripheral neuropathy among women with breast cancer in a Medicare population. Cancer, 2018

22. Dolan ME et al (2017) Clinical and Genome-Wide Analysis of Cisplatin-Induced Peripheral Neuropathy in Survivors of Adult-Onset Cancer. Clin Cancer Res 23(19):5757-5768

23. Yamaguchi $\mathrm{K}$ et al (2018) The risk factors for oxaliplatin-induced peripheral sensory neuropathy and thrombocytopenia in advanced gastric cancer. Cancer Chemother Pharmacol 82(4):625-633

24. Johnson C et al (2015) Candidate pathway-based genetic association study of platinum and platinum-taxane related toxicity in a cohort of primary lung cancer patients. Journal of the neurological sciences 349(1):124-128

25. Miltenburg NC, Boogerd W (2014) Chemotherapy-induced neuropathy: A comprehensive survey. Cancer Treat Rev 40(7):872-882

26. Wang XM et al (2012) Discovering cytokines as targets for chemotherapy-induced painful peripheral neuropathy. Cytokine 59(1):3-9

27. Lees JG et al (2017) Immune-mediated processes implicated in chemotherapy-induced peripheral neuropathy. Eur J Cancer 73:22-29 
28. Brandolini L et al., Chemokine Signaling in Chemotherapy-Induced Neuropathic Pain. Int J Mol Sci, 2019. 20(12)

29. Fumagalli $G$ et al (2020) Neuroinflammatory Process Involved in Different Preclinical Models of Chemotherapy-Induced Peripheral Neuropathy. Front Immunol 11:626687

30. Zhou L et al (2020) The Therapeutic Potential of Chemokines in the Treatment of ChemotherapyInduced Peripheral Neuropathy. Curr Drug Targets 21(3):288-301

31. Keswani SC et al (2003) Schwann cell chemokine receptors mediate HIV-1 gp120 toxicity to sensory neurons. Ann Neurol 54(3):287-296

32. Schafers M, Sorkin L (2008) Effect of cytokines on neuronal excitability. Neurosci Lett 437(3):188193

33. Makker PG et al (2017) Characterisation of Immune and Neuroinflammatory Changes Associated with Chemotherapy-Induced Peripheral Neuropathy. PLoS One 12(1):e0170814

34. Chen El et al (2015) Identifying Predictors of Taxane-Induced Peripheral Neuropathy Using Mass Spectrometry-Based Proteomics Technology. PLoS One 10(12):e0145816

35. Kanbayashi $Y$ et al (2010) Statistical identification of predictors for peripheral neuropathy associated with administration of bortezomib, taxanes, oxaliplatin or vincristine using ordered logistic regression analysis. Anticancer Drugs 21(9):877-881

36. Yamanouchi $\mathrm{K}$ et al (2017) The relationship between peripheral neuropathy induced by docetaxel and systemic inflammation-based parameters in patients with breast cancer. Anticancer research 37(12):6947-6951

37. Furberg CD, Friedman LM (2012) Approaches to data analyses of clinical trials. Prog Cardiovasc Dis 54(4):330-334

38. Kleckner IR et al (2018) Effects of exercise during chemotherapy on chemotherapy-induced peripheral neuropathy: a multicenter, randomized controlled trial. Support Care Cancer 26(4):10191028

39. Kleckner IR et al (2018) Response to Crevenna and Ashbury, Vallance and Bolam, and Crevenna and Keilani regarding the effects of exercise on chemotherapy-induced peripheral neuropathy. Support Care Cancer

40. Loh KP et al (2019) Effects of a Home-based Exercise Program on Anxiety and Mood Disturbances in Older Adults with Cancer Receiving Chemotherapy. J Am Geriatr Soc 67(5):1005-1011

41. Kleckner IR et al (2019) Effects of exercise on inflammation in patients receiving chemotherapy: a nationwide NCORP randomized clinical trial. Support Care Cancer 27(12):4615-4625

42. Marcus BH et al (1992) Self-efficacy and the stages of exercise behavior change. Res Q Exerc Sport 63(1):60-66

43. Mendoza TR et al (2013) The validity and utility of the M. D. Anderson Symptom Inventory in patients with breast cancer: evidence from the symptom outcomes and practice patterns data from the eastern cooperative oncology group. Clin Breast Cancer 13(5):325-334 
44. Smets EM et al (1995) The Multidimensional Fatigue Inventory (MFI) psychometric qualities of an instrument to assess fatigue. J Psychosom Res 39(3):315-325

45. Radloff LS (1977) The CES-D scale: A self report depression scale for research in the general population. Applied Psychological Measurements 1:385-401

46. Hann D, Winter K, Jacobsen P (1999) Measurement of depressive symptoms in cancer patients: evaluation of the Center for Epidemiological Studies Depression Scale (CES-D). J Psychosom Res 46(5):437-443

47. Spielberger C (1983) Manual for the State-Trait Anxiety Inventory. Consulting Psychologists Press, Palo Alto

48. Korfage IJ et al (2006) Anxiety and depression after prostate cancer diagnosis and treatment: 5-year follow-up. Br J Cancer 94(8):1093-1098

49. Bender R, Lange S (2001) Adjusting for multiple testing-when and how? J Clin Epidemiol 54:343349

50. Heinze G, Wallisch C, Dunkler D (2018) Variable selection - A review and recommendations for the practicing statistician. Biom J 60(3):431-449

51. Scheller $J$ et al (2011) The pro- and anti-inflammatory properties of the cytokine interleukin-6. Biochim Biophys Acta 1813(5):878-888

52. Al-Mazidi S et al (2018) Blocking of cytokines signalling attenuates evoked and spontaneous neuropathic pain behaviours in the paclitaxel rat model of chemotherapy-induced neuropathy. Eur $J$ Pain 22(4):810-821

53. Peila E et al (2016) Chemotherapy-Induced Neurotoxicity: Evidence of a Protective Role of CC Homozygosis in the Interleukin-1 beta Gene-511 C > T Polymorphism. Neurotox Res 30(3):521-529

54. Miaskowski C et al (2019) Perturbations in neuroinflammatory pathways are associated with paclitaxel-induced peripheral neuropathy in breast cancer survivors. J Neuroimmunol 335:577019

55. Ma J et al (2018) Beyond symptomatic relief for chemotherapy-induced peripheral neuropathy: Targeting the source. Cancer 124(11):2289-2298

56. Ma J et al (2018) Beyond symptomatic relief for chemotherapy-induced peripheral neuropathy: Targeting the source. Cancer 124(11):2289-2298

57. Hu L-Y et al (2019) Prevention and treatment for chemotherapy-induced peripheral neuropathy: therapies based on CIPN mechanisms. Current neuropharmacology 17(2):184-196

58. McLeary F et al (2019) Mechanisms underlying select chemotherapeutic-agent-induced neuroinflammation and subsequent neurodegeneration. Eur J Pharmacol 842:49-56

59. Nudelman $\mathrm{KN}$ et al (2016) Cerebral perfusion and gray matter changes associated with chemotherapy-induced peripheral neuropathy. Journal of clinical oncology 34(7):677

60. Ganz PA, Dougherty PM (2016) Painful hands and feet after cancer treatment: Inflammation affecting the mind-body connection. American Society of Clinical Oncology 
61. Omran M et al., Review of the role of the brain in chemotherapy-induced peripheral neuropathy $(C I P N)$. Frontiers in Molecular Biosciences, in revision

62. Kleckner IR et al., Evidence for a Large-Scale Brain System Supporting Allostasis and Interoception in Humans. Nat Hum Behav, 2017. 1

63. Vichaya EG et al (2015) Mechanisms of chemotherapy-induced behavioral toxicities. Front Neurosci 9:131

64. Raison CL, Capuron L, Miller AH (2006) Cytokines sing the blues: inflammation and the pathogenesis of depression. Trends Immunol 27(1):24-31

65. Ji YB et al (2017) Association of Inflammatory Cytokines With the Symptom Cluster of Pain, Fatigue, Depression, and Sleep Disturbance in Chinese Patients With Cancer. J Pain Symptom Manage 54(6):843-852

66. Kleckner IR et al (2021) Systematic review of exercise for prevention and managment of chemotherapy-induced peripheral neuropathy in. In: Lustberg MB, Loprinzi C Diagnosis, Management, and Emerging Strategies for Chemotherapy Induced Neuropathy. Springer, Editors

67. Wegner $\mathrm{M}$ et al (2014) Effects of exercise on anxiety and depression disorders: review of metaanalyses and neurobiological mechanisms. CNS Neurol Disord Drug Targets 13(6):1002-1014

68. Mustian KM et al (2017) Comparison of Pharmaceutical, Psychological, and Exercise Treatments for Cancer-Related Fatigue: A Meta-analysis. JAMA Oncol 3(7):961-968

69. Gleeson $\mathrm{M}$ et al (2011) The anti-inflammatory effects of exercise: mechanisms and implications for the prevention and treatment of disease. Nat Rev Immunol 11(9):607-615

70. Williamson JW et al (1997) Activation of the insular cortex during dynamic exercise in humans. $J$ Physiol 503(Pt 2):277-283

71. Kleckner IR et al (2018) Exercise for Toxicity Management in Cancer-A Narrative Review. Oncol Hematol Rev 14(1):28-37

72. Duregon $\mathrm{F}$ et al (2018) Effects of exercise on cancer patients suffering chemotherapy-induced peripheral neuropathy undergoing treatment: A systematic review. Crit Rev Oncol Hematol 121:90100

73. Kanzawa-Lee GA et al (2020) Exercise Effects on Chemotherapy-Induced Peripheral Neuropathy: A Comprehensive Integrative Review. Cancer Nurs 43(3):E172-E185

74. Goldstein DJ et al (2002) Duloxetine in the treatment of major depressive disorder: a double-blind clinical trial. J Clin Psychiatry 63(3):225-231

75. Smith EM et al (2013) Effect of duloxetine on pain, function, and quality of life among patients with chemotherapy-induced painful peripheral neuropathy: a randomized clinical trial. JAMA 309(13):1359-1367

76. MacKenzie MB, Abbott KA, Kocovski NL (2018) Mindfulness-based cognitive therapy in patients with depression: current perspectives. Neuropsychiatr Dis Treat 14:1599-1605 
77. Hofmann SG et al (2012) The Efficacy of Cognitive Behavioral Therapy: A Review of Meta-analyses. Cognit Ther Res 36(5):427-440

78. Al-Harbi KS, Qureshi NA (2012) Neuromodulation therapies and treatment-resistant depression. Med Devices (Auckl) 5:53-65

79. Prinsloo $S$ et al (2017) Randomized controlled trial of neurofeedback on chemotherapy-induced peripheral neuropathy: A pilot study. Cancer 123(11):1989-1997

80. Heisler M et al (2007) Mechanisms for racial and ethnic disparities in glycemic control in middleaged and older Americans in the health and retirement study. Arch Intern Med 167(17):1853-1860

81. Karter AJ et al (2007) Educational disparities in health behaviors among patients with diabetes: the Translating Research Into Action for Diabetes (TRIAD) Study. BMC Public Health 7:308

82. Menke A et al (2015) Prevalence of and Trends in Diabetes Among Adults in the United States, 1988-2012. JAMA 314(10):1021-1029

83. Loprinzi CL et al (2011) Natural history of paclitaxel-associated acute pain syndrome: prospective cohort study NCCTG N08C1. J Clin Oncol 29(11):1472-1478

\section{Tables}


Table 1

Patient baseline characteristics.

\begin{tabular}{|c|c|c|}
\hline Sample Characteristic & All & $\begin{array}{l}\text { Patients who provided } \\
\text { blood }\end{array}$ \\
\hline Total participants & 116 & 55 \\
\hline Age, years $(M \pm S D)$ & $55.4 \pm 9.8$ & $56.7 \pm 9.1$ \\
\hline Body mass index, $\mathrm{kg} / \mathrm{m}^{2}(\mathrm{M} \pm \mathrm{SD})$ & $30.3 \pm 6.1$ & $29.7 \pm 6.1$ \\
\hline Daily steps from pedometer $(\mathrm{M} \pm \mathrm{SD})$ & $\begin{array}{l}4,412 \pm \\
2961\end{array}$ & $4,695 \pm 3,192$ \\
\hline $\begin{array}{l}\text { Time since end of first radiation or surgery for cancer, } \\
\text { weeks }(M \pm S D)\end{array}$ & $6.4 \pm 12.4$ & $4.9 \pm 7.0$ \\
\hline \multicolumn{3}{|l|}{ Race } \\
\hline White & $95(82 \%)$ & $46(84 \%)$ \\
\hline Black & $14(12 \%)$ & $7(13 \%)$ \\
\hline Other & $7(6 \%)$ & $2(4 \%)$ \\
\hline \multicolumn{3}{|l|}{ Employment } \\
\hline Employed outside the house & $64(55 \%)$ & $35(64 \%)$ \\
\hline Unemployed & $39(34 \%)$ & $15(27 \%)$ \\
\hline Self-employed / homemaker & $13(11 \%)$ & $5(9 \%)$ \\
\hline \multicolumn{3}{|l|}{ Marital status } \\
\hline Married or long-term committed relationship & $76(66 \%)$ & $31(56 \%)$ \\
\hline Divorced, separated, single, widowed & $40(34 \%)$ & $24(44 \%)$ \\
\hline \multicolumn{3}{|l|}{ Education } \\
\hline At least some college & $78(67 \%)$ & $34(62 \%)$ \\
\hline High school/GED degree & $33(28 \%)$ & $18(33 \%)$ \\
\hline No high school or GED degree & $4(3 \%)$ & $2(4 \%)$ \\
\hline Unknown & $1(1 \%)$ & $1(2 \%)$ \\
\hline \multicolumn{3}{|l|}{ Cancer stage } \\
\hline Stage I & $41(35 \%)$ & $20(36 \%)$ \\
\hline Stage II & 62 (53\%) & 31 (56\%) \\
\hline
\end{tabular}




\begin{tabular}{|lll|}
\hline Sample Characteristic & All & $\begin{array}{l}\text { Patients who provided } \\
\text { blood }\end{array}$ \\
\hline Stage III & $12(10 \%)$ & $4(7 \%)$ \\
\hline Stage IV & $0(0 \%)$ & $0(0 \%)$ \\
\hline Unknown & $1(1 \%)$ & $0(0 \%)$ \\
\hline Previous treatment & $105(91 \%)$ & $50(91 \%)$ \\
\hline Previous surgery & $2(2 \%)$ & $2(4 \%)$ \\
\hline Previous hormone therapy & $2(2 \%)$ & $1(2 \%)$ \\
\hline Previous radiation therapy & $81(70 \%)$ & $42(76 \%)$ \\
\hline Neurotoxic chemotherapy type & $35(30 \%)$ & $13(24 \%)$ \\
\hline Taxane-based only & $10(9 \%)$ & $7(13 \%)$ \\
\hline Platinum- and taxane-based & $37(32 \%)$ & $18(33 \%)$ \\
\hline Karnofsky Performance Status & $69(59 \%)$ & $30(55 \%)$ \\
\hline 80 & & \\
\hline 90 & & \\
\hline 100 & & \\
\hline
\end{tabular}


Table 2

Patient-reported severity of neuropathy symptoms before and after 6 weeks of neurotoxic chemotherapy.

\begin{tabular}{|c|c|c|c|c|c|c|c|}
\hline & \multicolumn{2}{|c|}{$\begin{array}{l}\text { Before } \\
\text { chemotherapy }\end{array}$} & \multicolumn{2}{|c|}{$\begin{array}{l}\text { After } 6 \text { weeks of } \\
\text { chemotherapy }\end{array}$} & \multicolumn{3}{|c|}{$\begin{array}{l}\text { Change (after minus } \\
\text { before) }\end{array}$} \\
\hline & Mean & $\begin{array}{l}95 \% \\
\mathrm{Cl}\end{array}$ & Mean & $95 \% \mathrm{Cl}$ & Mean & $\begin{array}{l}95 \% \\
\mathrm{Cl}\end{array}$ & $\begin{array}{l}p- \\
\text { value }\end{array}$ \\
\hline \multicolumn{8}{|l|}{ Sample: all 116 patients } \\
\hline $\begin{array}{l}\text { Severity of numbness and tingling } \\
\text { in the past week }(0-10)\end{array}$ & 1.14 & $\begin{array}{l}0.76 \\
1.52\end{array}$ & 1.78 & $\begin{array}{l}1.34 \\
2.21\end{array}$ & 0.64 & $\begin{array}{l}0.17 \\
1.11\end{array}$ & 0.0089 \\
\hline $\begin{array}{l}\text { Severity of hot/coldness in } \\
\text { hand/feet in the past week }(0-10)\end{array}$ & 0.92 & $\begin{array}{l}0.56 \\
1.29\end{array}$ & 1.64 & $\begin{array}{l}1.16 \\
2.11\end{array}$ & 0.72 & $\begin{array}{l}0.29 \\
1.14\end{array}$ & 0.0013 \\
\hline \multicolumn{8}{|l|}{$\begin{array}{l}\text { Sample: all } 55 \text { patients who } \\
\text { provided blood }\end{array}$} \\
\hline $\begin{array}{l}\text { Severity of numbness and tingling } \\
\text { in the past week }(0-10)\end{array}$ & 0.98 & $\begin{array}{l}0.48 \\
1.48\end{array}$ & 1.71 & $\begin{array}{l}\text { 1.07, } \\
2.35\end{array}$ & 0.73 & $\begin{array}{l}0.18 \\
1.28\end{array}$ & 0.0124 \\
\hline $\begin{array}{l}\text { Severity of hot/coldness in } \\
\text { hand/feet in the past week }(0-10)\end{array}$ & 1.07 & $\begin{array}{l}0.47 \\
1.67\end{array}$ & 1.84 & $\begin{array}{l}1.08 \\
2.59\end{array}$ & 0.76 & $\begin{array}{l}0.17 \\
1.35\end{array}$ & 0.0139 \\
\hline
\end{tabular}

Table 3

Patient-reported prevalence of neuropathy symptoms before and after 6 weeks of neurotoxic chemotherapy.

\section{Before After 6 weeks of Change (after chemotherapy chemotherapy minus before)}

Sample: all 116 patients

Prevalence of numbness and tingling in $36 \% \quad 53 \% \quad 17 \%$

the past week $(1-10)$

Prevalence of hot/coldness in hand/feet $\quad 27 \% \quad 43 \% \quad 16 \%$

in the past week $(1-10)$

Sample: all 55 patients who provided

blood

Prevalence of numbness and tingling in

the past week $(1-10)$

$36 \%$

$51 \%$

$15 \%$

Prevalence of hot/coldness in hand/feet in the past week $(1-10)$

$27 \%$

$42 \%$

$15 \%$ 
Table 4

Model fit using stepwise regression for outcome of patient-reported severity of neuropathy symptoms in all 116 patients before and after 6 weeks of neurotoxic chemotherapy.

\begin{tabular}{|c|c|c|c|c|}
\hline Term $^{a}$ & $\begin{array}{l}\text { Estimate } \\
\text { b }\end{array}$ & $95 \% \mathrm{Cl}$ & p-value & $\eta_{p}^{2}$ \\
\hline \multicolumn{5}{|l|}{$\begin{array}{l}\text { Outcome: Severity of numbness and tingling in the past } \\
\text { week }(0-10)\end{array}$} \\
\hline Intercept & -1.98 & $\begin{array}{l}-4.11 \\
0.15\end{array}$ & 0.068 & - \\
\hline Composite of Fatigue, Anxiety, Depression & 1.32 & $\begin{array}{l}0.91 \\
1.72\end{array}$ & $<.0001$ & $27.0 \%$ \\
\hline Age (years) & 0.06 & $\begin{array}{l}0.02 \\
0.10\end{array}$ & 0.002 & $8.5 \%$ \\
\hline Baseline neuropathy (numbness \& tingling) & 0.23 & $\begin{array}{l}0.05 \\
0.41\end{array}$ & 0.014 & $5.3 \%$ \\
\hline Race (black minus other) & 0.97 & $\begin{array}{l}-0.14 \\
2.08\end{array}$ & 0.085 & $2.6 \%$ \\
\hline \multicolumn{5}{|l|}{$\begin{array}{l}\text { Outcome: Severity of hot/coldness in hands/feet in the } \\
\text { past week }(0-10)\end{array}$} \\
\hline Intercept & 0.78 & $\begin{array}{l}0.01 \\
1.55\end{array}$ & 0.048 & - \\
\hline Baseline neuropathy (hot/coldness) & 0.47 & $\begin{array}{l}0.21 \\
0.73\end{array}$ & 0.0004 & $10.6 \%$ \\
\hline Composite of Fatigue, Anxiety, Depression & 0.71 & $\begin{array}{l}0.17 \\
1.24\end{array}$ & 0.010 & $5.8 \%$ \\
\hline Chemotherapy type (Taxane minus Taxane + Platinum) & 0.58 & $\begin{array}{l}-0.29 \\
1.46\end{array}$ & 0.190 & $1.5 \%$ \\
\hline
\end{tabular}


Table 5

Model fit using stepwise regression for outcome of patient-reported severity of neuropathy symptoms in all 55 patients who provided a blood sample before and after 6 weeks of neurotoxic chemotherapy.

\begin{tabular}{|c|c|c|c|c|}
\hline Term $^{a}$ & $\begin{array}{l}\text { Estimate } \\
\text { b }\end{array}$ & $95 \% \mathrm{Cl}$ & $\begin{array}{l}\mathrm{p}- \\
\text { value }\end{array}$ & $\mathrm{n}_{\mathrm{p}}^{2}$ \\
\hline \multicolumn{5}{|c|}{$\begin{array}{l}\text { Outcome: Severity of numbness and tingling in the past } \\
\text { week }(0-10)\end{array}$} \\
\hline Intercept & 4.35 & $\begin{array}{l}1.01 \\
7.68\end{array}$ & 0.012 & - \\
\hline Baseline neuropathy (numbness \& tingling) & 0.55 & $\begin{array}{l}0.28 \\
0.81\end{array}$ & 0.0001 & $27.9 \%$ \\
\hline Cancer stage ( 1 minus 2,3 , and 4 ) & 1.48 & $\begin{array}{l}0.51 \\
2.44\end{array}$ & 0.004 & $17.4 \%$ \\
\hline Composite of Fatigue, Anxiety, Depression & 0.65 & $\begin{array}{l}0.18 \\
1.11\end{array}$ & 0.007 & $15.0 \%$ \\
\hline IFN-y (high minus low) & 1.16 & $\begin{array}{l}0.23 \\
2.09\end{array}$ & 0.016 & $12.3 \%$ \\
\hline Using diabetes medications & 1.97 & $\begin{array}{l}0.30 \\
3.64\end{array}$ & 0.022 & $11.2 \%$ \\
\hline Race (black minus other) & 1.40 & $\begin{array}{l}0.00 \\
2.79\end{array}$ & 0.050 & $8.3 \%$ \\
\hline IL-1 $\beta$ (high and low minus very low) & 0.42 & $\begin{array}{l}-0.07 \\
0.92\end{array}$ & 0.093 & $6.1 \%$ \\
\hline IL-10 & -1.33 & $\begin{array}{l}-2.96 \\
0.29\end{array}$ & 0.105 & $5.7 \%$ \\
\hline IL-8 & 0.44 & $\begin{array}{l}-1.13 \\
2.02\end{array}$ & 0.573 & $0.7 \%$ \\
\hline \multicolumn{5}{|c|}{$\begin{array}{l}\text { Outcome: Severity of hot/coldness in hands/feet in the } \\
\text { past week }(0-10)\end{array}$} \\
\hline Intercept & 6.46 & $\begin{array}{l}2.65 \\
10.26\end{array}$ & 0.001 & - \\
\hline Composite of Fatigue, Anxiety, Depression & 1.37 & $\begin{array}{l}0.66 \\
2.09\end{array}$ & 0.0004 & $26.3 \%$ \\
\hline IFN-y (high minus low) & 1.74 & $\begin{array}{l}0.56 \\
2.91\end{array}$ & 0.005 & $17.5 \%$ \\
\hline Baseline neuropathy (hot/coldness) & 0.46 & $\begin{array}{l}0.10 \\
0.82\end{array}$ & 0.014 & $13.5 \%$ \\
\hline
\end{tabular}



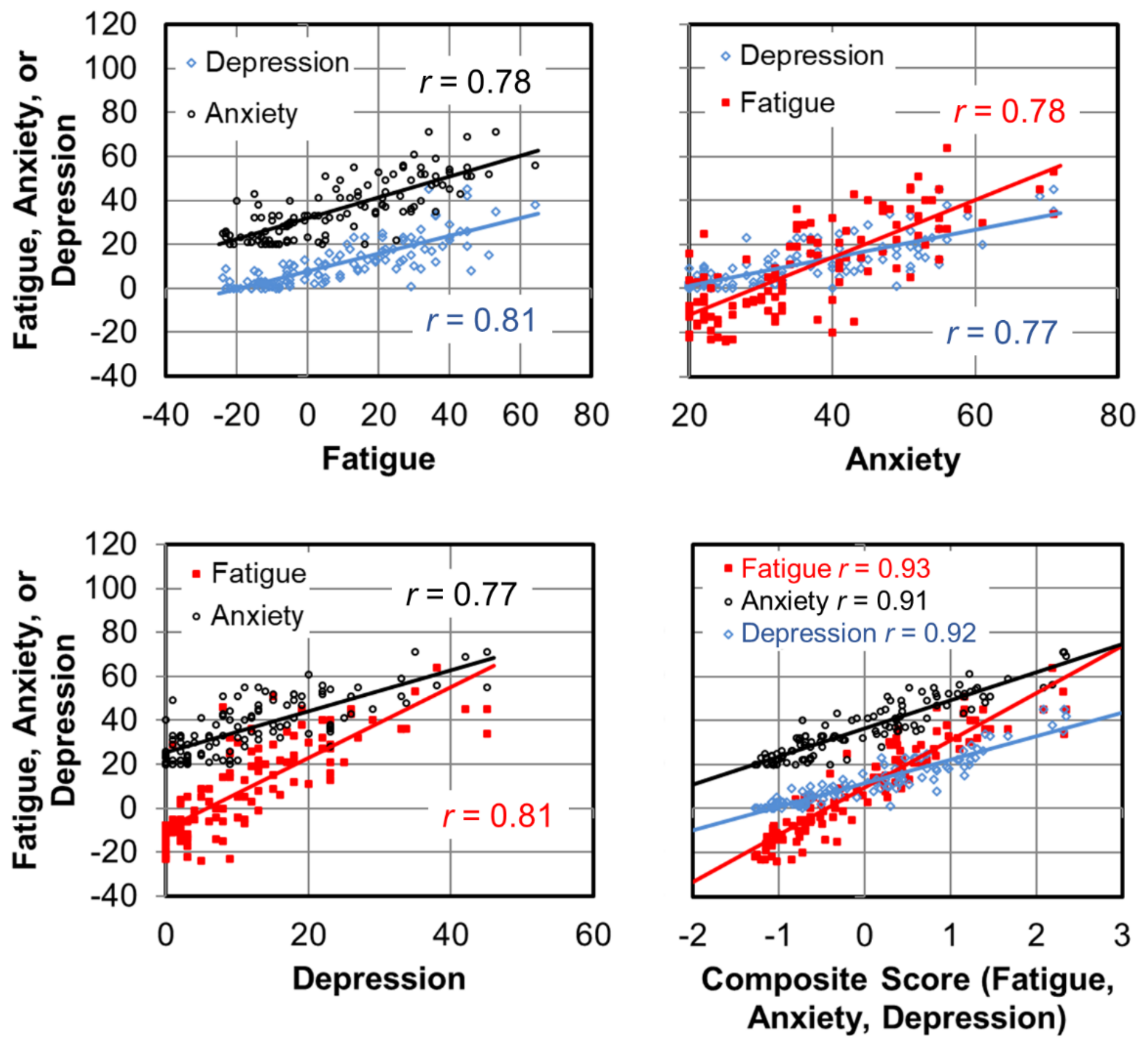

Figure 1

Patient-reported fatigue (MFSI), anxiety (STAI), and depression (CES-D) were all highly related via Person's correlation and thus were standardized and averaged into a single composite score. Each data point is from one of the 116 patients before starting neurotoxic chemotherapy. 

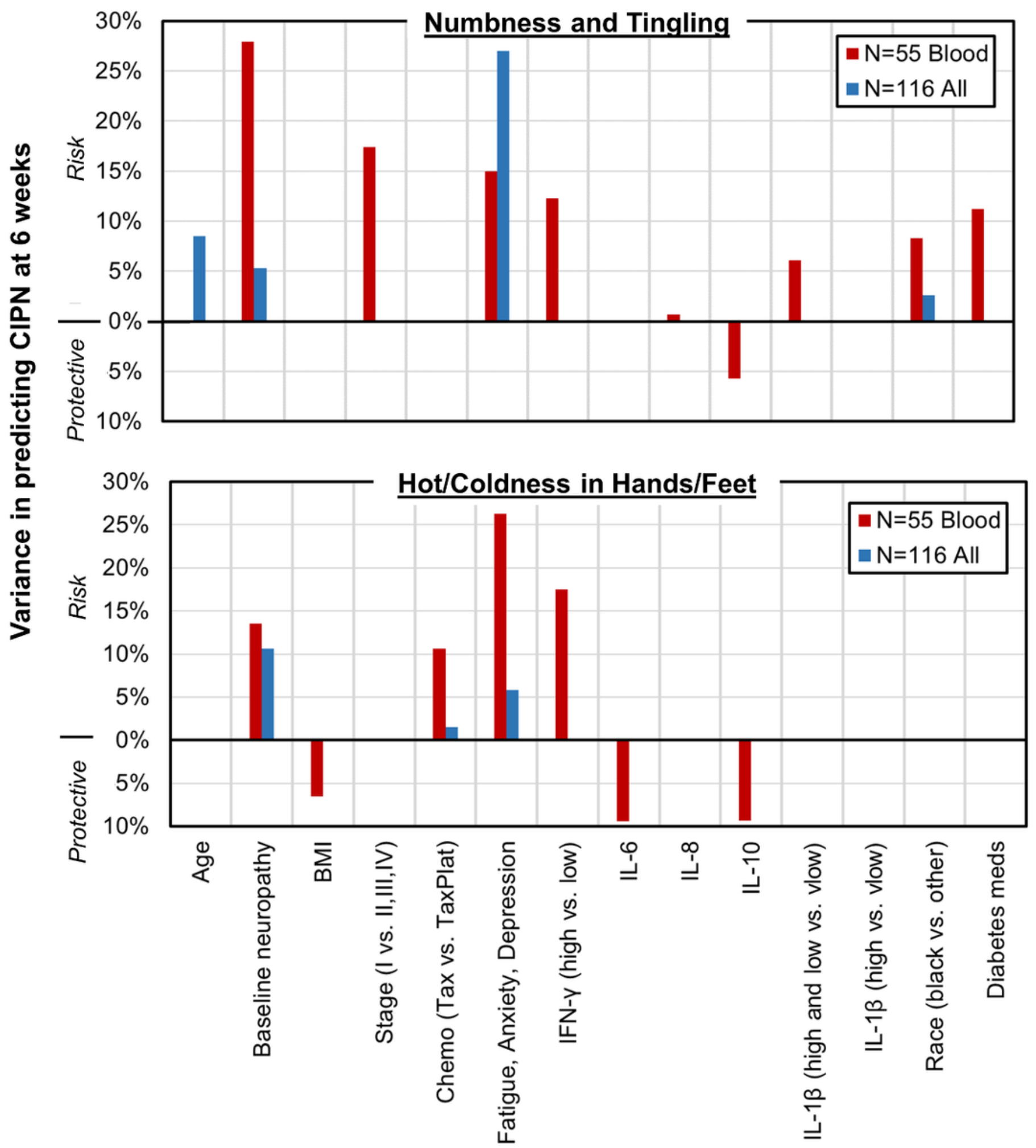

Figure 2

Variance explained for each potential risk factor in explaining numbness/tingling (top) or hot/coldness in hands/feet (bottom) for the two sub-cohorts. Risk factors are shown with percentage variance pointing upwards. Protective factors are shown with percentage variance pointing downwards. 